$$
\text { "abboud" — 2008/9/1 — 18:51 — page } 187 \text { — } \#
$$

\title{
Comment les enseignants en formation initiale utilisent les technologies informatiques dans leurs classes
}

\author{
Maha Abboud-Blanchard, Jean-Paul Fallot, Agnès Lenfant et \\ BERNARD PARZYSZ
}

\begin{abstract}
The research presented here deals with the way French pre-service teachers assimilate the working of technology tools and the effects on professional practice of integrating these tools into classes. We focused on the professional writings of pre-service teachers regarding the use of technology in their teaching. The results show that, besides official instructions, the motivations put forward by pre-service teachers who integrated technology in their classes are mainly their students' interest in computers and how powerful this tool is. They also show that in such an environment teachers tend to keep in the background and to leave the students to interact chiefly with the computer. We also noticed that the specificities of managing a classroom in computer environment are not taken into account unless they generate problems.

Résumé. La recherche présentée ici porte sur l'appropriation des outils informatiques par les enseignants français en formation initiale et les effets de leur intégration dans les classes sur les pratiques professionnelles. Nous avons pris comme objet d'étude des écrits professionnels, élaborés par ces professeurs stagiaires, portant sur l'utilisation des TIC dans leur enseignement. Les résultats obtenus font apparaître qu'outre les injonctions institutionnelles, les motivations invoquées par les stagiaires pour recourir à l'informatique concernent surtout l'attrait de leurs élèves pour l'ordinateur et la puissance de cet outil. Dans le cadre des usages en classe, nos résultats montrent que l'enseignant a tendance à s'effacer devant l'ordinateur, considéré comme l'interlocuteur privilégié de l'élève. Nous avons aussi pu constater que les spécificités de la gestion de la classe en environnement informatique ne sont prises en compte que lorsqu'elles se révèlent sources de problèmes.
\end{abstract}

Key words and phrases: pre-service teachers, professional dissertation, technology environment, teachers' behaviour and role in classroom.

ZDM Subject Classification: B10, B50, D40, U70.

Copyright (c) 2008 by University of Debrecen 
"abboud" — 2008/9/1 — 18:51 — page 188 — \#2

\section{Introduction}

On observe actuellement en France des incitations de plus en plus pressantes à l'usage des technologies dans l'enseignement. D'une part, l'institution a inscrit l'utilisation des $\mathrm{TICE}^{1}$ dans les programmes scolaires [14], [15 ${ }^{2}$ et dans le cursus de formation universitaire $[16]^{3}$. D'autre part, le taux d'équipement des établissements scolaires est maintenant globalement élevé, même s'il subsiste des difficultés, notamment au niveau de l'école primaire [17]. De plus, un nombre important de recherches ont montré les potentialités de l'usage des technologies informatiques pour l'apprentissage des élèves et pour un renouvellement des pratiques scolaires. Pourtant, malgré ces facteurs a priori encourageants, l'usage des technologies dans les classes, avec les élèves, n'est pas encore généralisé [7], [19]. Un rapport de l'OCDE s'appuyant sur des données issues de 14 pays membres, dont la France, indique que l'utilisation de l'informatique au quotidien dans les établissements reste décevante [25].

En amont de ces facteurs se posent la question des pratiques des enseignants dans ces environnements technologiques et celle de leur formation aux usages des TICE [1]. En effet, la "dimension" enseignant dans l'intégration des outils informatiques est, encore aujourd'hui, peu étudiée. Une méta-étude [20] souligne que "jusqu'en 1998, la tendance était de considérer implicitement que la diffusion des situations innovantes utilisant les technologies, parfois agrémentées de quelques résultats de recherches, fournit à l'enseignant le matériel nécessaire à une intégration aisée de ces technologies dans son enseignement" (op. cit. p. 257). La prise en compte de la complexité du travail de l'enseignant dans ces environnements, ainsi que la nécessité d'étudier les pratiques TICE des enseignants [24] sont des préoccupations relativement récentes. Pour notre part, nous abordons cette

1 Technologies de l'Information et de la Communication pour l'Enseignement.

2 Dans l'enseignement français, tous les élèves reçoivent des formations disciplinaires impliquant les TIC devant aboutir à l'obtention d'un Brevet Informatique et Internet (B2i).

${ }^{3}$ Le Certificat Informatique et Internet (C2i) comporte deux niveaux. Le premier atteste la maîtrise d'un ensemble de compétences techniques nécessaires pour mener les activités qu'exige aujourd'hui un cursus d'enseignement supérieur (http://c2i.education.fr). Le second atteste de compétences professionnelles communes et nécessaires à tous les enseignants pour l'exercice de leur métier, dans les dimensions pédagogique, éducative et citoyenne.

4 There was a tendency to focus on teachers' development and an implicit assumption that the transfer of innovative situations of use, possibly supported by outcomes of research, would provide the teacher with sufficient material for an easy integration. 
"abboud" — 2008/9/1 — 18:51 — page $189-\# 3$

dimension par un travail de recherche portant sur les enseignants en formation initiale à l'IUFM ${ }^{5}$.

Nous faisons l'hypothèse que les obstacles constatés et persistants à une généralisation de l'utilisation des TICE dans les disciplines sont à chercher plus du côté des pratiques émergentes en classe que du côté des compétences et pratiques développées hors classe. En effet, les enseignants débutants constituent un public mieux outillé et connecté que l'ensemble de la population [8], [5] pour lequel le recours aux technologies devrait être facilité et ne viendrait pas perturber leur intégration dans l'enseignement. De plus, plusieurs recherches sur les enseignants en formation ont montré que les pratiques professionnelles se stabilisent très rapidement au cours de l'année de stage [23], [9].

Dans la suite de l'article, nous commencerons par présenter le contexte et les questions de la recherche ainsi que nos cadres théoriques, puis nous détaillerons nos données et nos outils méthodologiques et nous terminerons par l'exposé de nos résultats ainsi que des nouvelles questions de recherche qui se posent à nous à l'issue de ce travail.

\section{Contexte et questions de la recherche}

La recherche décrite dans cet article a été menée dans le cadre d'un projet plus vaste dont le but était d'une part d'étudier les compétences acquises au sein de la formation universitaire et à l'IUFM ainsi que leurs limites; et d'autre part d'identifier et d'analyser les usages des technologies que les professeurs stagiaires intègrent le plus fréquemment dans leur enseignement, avec les points de résistance et les déterminants qui les sous-tendent [3]. Pour mener cette étude, trois cadres d'usages des technologies ont été distingués (ibid.) :

\footnotetext{
${ }^{5}$ Dans le système de formation français, l'accès au métier de professeur des écoles (PE) comme de professeur de lycées et collèges (PLC) est soumis à la réussite d'un concours auquel peuvent se présenter les titulaires d'une licence. Les IUFM (Instituts Universitaires de Formation des Maîtres) sont chargés de la formation professionnelle des candidats admis au concours, qui deviennent alors professeurs stagiaires pendant un an (PE2 ou PLC2). La formation professionnelle initiale de ces enseignants, fondée sur l'articulation entre la théorie et la pratique, repose sur deux dispositifs : des formations à l'IUFM et des stages en responsabilité en établissement scolaire (ces derniers diffèrent selon les filières). Au cours de leurs stages en responsabilité, les professeurs stagiaires sont accompagnés par des maîtres de stage et des formateurs IUFM, dans le cadre de dispositifs comportant un suivi et une évaluation propres à chaque institut.
} 
"abboud" — 2008/9/1 — 18:51 — page $190-\# 4$

- le premier concerne les activités professionnelles non directement liées à la classe, qu'elles s'exercent individuellement ou au sein de communautés enseignantes. Un grand nombre d'usages y sont possibles : communication avec des collègues, recherche d'information via l'Internet, production de ressources numériques ;

- le second cadre est celui où le professeur travaille "en différé" aux apprentissages de ses élèves (back-office). La conception de situations ou d'activités destinées aux élèves, la production de documents pour la classe, l'élaboration de fiches de préparation, l'évaluation des apprentissages sont des usages possibles dans ce cadre;

- le troisième cadre est celui de la classe. Les usages y ont pour objectif de soutenir des apprentissages disciplinaires en tirant parti de logiciels spécifiques à la discipline ou de logiciels généraux.

Une étude par questionnaires a d'abord été réalisée [22]. L'analyse des réponses obtenues confirme un taux d'équipement informatique élevé chez les professeurs stagiaires. Les compétences qu'ils déclarent font apparaître une maîtrise de l'édition de textes courts et de la recherche d'informations sur l'Internet. Relativement au deuxième cadre d'usage, la majorité des professeurs stagiaires utilise l'Internet pour y rechercher des idées et des ressources, édite leurs fiches de préparation et les documents élèves à l'aide d'un logiciel de traitement de texte. L'utilisation de logiciels disciplinaires pour la préparation de la classe est minoritaire chez les PE et très variable suivant les disciplines chez les PLC. En ce qui concerne le troisième cadre d'usage, les stagiaires se déclarent prêts, si les conditions matérielles le permettent, à utiliser les TICE en classe, car ils y voient plusieurs intérêts : sensibilisation des élèves aux technologies, contribution des logiciels à l'enseignement disciplinaire et aide à la motivation des élèves.

Dans un second temps a été entreprise une étude - qui constitue le sujet de cet article - visant à préciser les pratiques réelles des professeurs stagiaires à travers l'analyse de leurs mémoires professionnels ${ }^{6}$. L'objectif principal était d'apporter des éléments de réponses à des questions relatives aux usages de l'ordinateur mis en oeuvre par les stagiaires dans leurs classes. Ces questions étaient issues des travaux antérieurs de notre équipe et/ou de questions rencontrées en formation :

\footnotetext{
${ }^{6}$ L'évaluation des professeurs stagiaires par l'IUFM prenait en compte, au moment de la réalisation de cette recherche, trois éléments : les stages en responsabilité, les modules d'enseignement dispensés à l'institut et le mémoire professionnel.
} 
"abboud" — 2008/9/1 — 18:51 — page 191 — \#5

Comment les enseignants en formation initiale utilisent l'informatique en classe

- Les apports de l'intégration des technologies dans l'enseignement mis en avant par les stagiaires sont-ils plutôt liés aux aspects disciplinaires ou à l'initiation et à la formation des élèves à ces technologies? Cette question se base essentiellement sur les enquêtes qui sont, depuis quelques années, menées en début de formation dans un certain nombre d'IUFM, en vue de piloter celle-ci [21].

- Y a-t-il des différences entre la préparation d'une séance intégrant un outil TICE et celle d'une séance papier/crayon? Le stagiaire prend-il en compte la gestion spécifique liée aux nouvelles formes de travail (comme la répartition des élèves en groupes autour d'ordinateurs ou l'utilisation interactive d'un poste unique avec vidéo-projection) et la spécificité conceptuelle des tâches rattachée aux "contenus embarqués" dans le logiciel? Ou bien les priorités prises en compte dans la préparation ne concernent-elles que la gestion matérielle et les tâches "techniques" liées à la manipulation du logiciel? Ces questions s'appuient sur les résultats de travaux portant sur les problématiques de l'intégration des TICE comme par exemple [4], [20].

- Dans les séances mises en ouvre par les stagiaires, existe-t-il des phases collectives, en particulier des moments de bilan? En effet, ces phases sont plus particulièrement susceptibles de faire apparaître une mise en relation des activités des élèves avec les savoirs à construire et une synthèse relative à la mise en œuvre des TICE [2].

\section{Cadres théoriques}

Ces dernières années, différents éléments tels que la mise en place d'un système éducatif de masse, la modification du public scolaire, l'avancée des connaissances dans le domaine de l'apprentissage et le développement des outils numériques de communication ont conduit à une évolution de la perception du métier d'enseignant. Cette évolution s'est accompagnée de la reconnaissance d'une spécificité de la professionnalité enseignante [27] et d'une réflexion sur la nature et la genèse des pratiques professionnelles [26]. Des travaux concernant plus particulièrement les environnements TICE soulignent qu'une professionnalité ayant ces environnements comme élément structurant nécessite que les enseignants ne pratiquent plus les TICE comme une dimension "isolée" mais considèrent que leur travail inclut une dimension instrumentale à part entière [13]. 
"abboud" — 2008/9/1 — 18:51 — page $192-\# 6$

Différents cadres théoriques ont été développés pour analyser les pratiques des enseignants. Pour notre recherche, nous nous appuyons plus particulièrement sur celui développé par Robert et Rogalski [29] : la double approche ergonomique et didactique des pratiques enseignantes. L'objectif de ce cadre théorique, s'appuyant sur des travaux issus de la psychologie ergonomique et de la didactique des mathématiques, est de contribuer à l'analyse et à la compréhension des pratiques des enseignants, tant du point de vue de ce qu'elles peuvent engendrer en termes d'apprentissages des élèves que du point de vue des impératifs professionnels auxquels elles répondent, par rapport à l'enseignant lui-même et pas seulement par rapport à ses élèves. Différentes recherches prenant appui sur ce cadre théorique ont permis d'approcher la complexité des pratiques professionnelles enseignantes et le fait qu'elles s'organisent en systèmes stables et cohérents. Lenfant [23] a, en particulier, mis en évidence que cette cohérence se construit rapidement chez les professeurs stagiaires d'IUFM et qu'elle se fige lors de l'année de stage, sous l'effet de représentations antérieures et de celles qui se forment dans la pratique. Selon Lenfant, sous l'influence des réalités du terrain et de la formation, certaines compétences sont plus rapides que d'autres à se développer, mais la mise en place de dispositifs de formation spécifiques peut contribuer à faire naître des questionnements qui ne se construisent pas a priori facilement.

Pour prendre en compte la spécificité des pratiques dans les environnements TICE, nous nous appuyons sur le cadre de l'approche instrumentale développé par Rabardel [28]. Il s'agit, dans cette approche, de distinguer l'objet ou artefact et l'instrument que cet objet devient au service de l'activité d'un individu donné ou d'une institution. La transition artefact-instrument se produit via une genèse instrumentale, en général complexe. Cette genèse met en jeu des processus d'instrumentalisation, dirigés vers l'artefact, et des processus d'instrumentation, dirigés vers le sujet. Ces derniers se traduisent par le développement de schèmes d'action instrumentée qui sont à la base des techniques instrumentées servant à la résolution des tâches proposées au sujet. Se référant à cette approche, Goigoux [18] définit les schèmes d'utilisation associés aux artefacts, dans l'activité de l'enseignant, comme résultant "d'une construction personnelle ou de l'appropriation de schèmes professionnels préexistants" (op. cit. p. 24). Il ajoute que ces schèmes ont une dimension privée, puisqu'ils sont propres à chaque individu, et qu'ils ont également une dimension sociale, c'est-à-dire qu'il convient de les considérer comme inscrits dans la mémoire impersonnelle du collectif professionnel. Ce cadre nous a permis en particulier d'étudier l'activité instrumentée des 


$$
\text { "abboud" — 2008/9/1 — 18:51 — page } 193 \text { — \#7 }
$$

enseignants stagiaires et les déterminants des genèses d'usages professionnels des TICE.

\section{Le mémoire professionnel, trace d'une pratique réelle des TICE}

L'analyse des réponses à des questionnaires (première entrée méthodologique utilisée dans notre projet) fournit certes des renseignements importants sur l'intégration des TICE par les stagiaires d'IUFM dans leurs classes, mais elle repose uniquement sur les déclarations des intéressés et peut par conséquent comporter des biais, voulus ou non; en particulier, on a pu constater une difficulté des stagiaires à bien se positionner par rapport à leurs compétences. Elle demandait donc à être complétée par une analyse des pratiques effectives en situation d'enseignement. Pour cela, nous avons choisi d'utiliser des "traces" [30] de pratiques en recourant à une observation indirecte à travers l'étude d'écrits professionnels, en l'occurrence des mémoires ayant pour sujet l'utilisation des TICE en classe. En tant que trace de pratiques, le mémoire professionnel présente en effet des caractéristiques intéressantes pour notre étude :

- son élaboration constitue un moment privilégié de la formation, au cours duquel le stagiaire met en place, en y consacrant suffisamment de temps et en appuyant sa réflexion sur la formation reçue comme sur la littérature existante, une séquence d'enseignement qu'il considère souvent comme "exemplaire";

- il décrit et analyse les éléments jugés par lui essentiels dans l'élaboration et la mise en œuvre de la séquence;

- il met par écrit son cheminement intellectuel, explicite les raisons de ses choix et les enseignements qu'il a tirés de cette expérience.

Cependant, toute méthodologie d'observation (directe ou indirecte) comportant des biais et des difficultés, il nous fallait ici tenir compte des conditions institutionnelles dans lesquelles ces documents ont été produits. En effet, le mémoire professionnel est par nature tiraillé entre deux pôles puisqu'il contribue à la constitution de la professionnalité du stagiaire et qu'il est également l'objet d'une validation institutionnelle. En outre, le rôle du tuteur s'avère fondamental dans son processus d'élaboration. Par ailleurs, le mémoire est réalisé dans des conditions variables d'un IUFM à l'autre, d'une discipline à l'autre et d'un tuteur à l'autre. A priori cette variabilité risque de ne pas permettre de dégager 


$$
\text { "abboud" — 2008/9/1 — 18:51 — page } 194-\# 8
$$

des constantes, mais a contrario, si des régularités apparaissent, elles seront révélatrices de jalons importants dans la genèse des pratiques professionnelles des stagiaires relativement aux TICE.

\section{Méthodologie}

Pour mener notre étude, nous avons choisi, à l'instar d'autres recherches portant sur les mémoires professionnels, d'utiliser une grille d'analyse. Les grilles existantes que nous avons pu consulter - en particulier [12] - ne nous ont pas paru exploitables car elles s'intéressent à une caractérisation du mémoire comme texte ou comme outil de formation, alors que pour nous il s'agissait d'étudier les usages qu'il rapporte et analyse. Nous avons donc élaboré une grille d'analyse des mémoires et l'avons ensuite fait fonctionner sur un corpus provenant des cinq IUFM participant à la recherche.

\section{Corpus et thèmes}

Nous nous sommes limités à deux années universitaires, afin d'assurer l'homogénéité du public et de la formation, et aux mémoires relatifs à des activités scientifiques $^{7}$.

Nous avons, dans un premier temps, retenu tous les mémoires dont le titre mentionnait explicitement les TICE. L'étude de ce premier corpus nous a conduits à répartir les mémoires de $\mathrm{PE}$ selon trois thèmes : 1- enseignement des sciences, 2- enseignement des mathématiques et 3-initiation à l'informatique en vue du B2i [15]. Pour ce qui est des PLC, nous avons retenu les six thèmes suivants : Asciences et techniques, B- géométrie, C- statistiques, D- algèbre en liaison avec la géométrie, $\mathrm{E}$ - tableurs dans les disciplines scientifiques (hors mathématiques) et $\mathrm{F}$ - initiation à l'informatique (en vue du B2i).

À l'issue de cette première phase, 72 mémoires (51 PLC et $21 \mathrm{PE}$ ) ont été retenus. Puis, pour obtenir un équilibre entre les divers thèmes et les divers IUFM, nous avons opéré une nouvelle sélection, qui nous a fourni un corpus définitif de 28 mémoires (22 PLC et $6 \mathrm{PE}$ ). Cette sélection est telle que, dans chaque thème, les mémoires retenus permettent une première analyse du type d'activités TICE

7 L'étude des mémoires supposant des entrées didactiques, seules celles relatives aux disciplines scientifiques étaient réellement accessibles aux membres de l'équipe. 


$$
\text { "abboud" — 2008/9/1 — 18:51 — page } 195 \text { — \#9 }
$$

qui peuvent être menées, ainsi qu'un accès à la réflexion que peut développer un professeur stagiaire sur ces activités.

\section{La grille d'analyse}

La grille élaborée comprend un ensemble complet d'indicateurs qualitatifs relatifs aux usages possibles et correspondant à nos hypothèses. Dans le but de faciliter le travail d'analyse, l'enchaînement des questions de la grille suit approximativement la structure usuelle du mémoire : informations d'ordre général, problématique et analyse a priori (lorsqu'elle existe), compte rendu et analyse du travail effectif, conclusion.

La grille est ainsi composée de quatre parties, présentées succinctement ici. Ses différents items sont directement issus des questions de la recherche présentées plus haut. Nous renvoyons le lecteur en annexe pour consulter l'intégralité de la grille.

La partie 1 traite des identifiants du mémoire (niveau(x) de classe, nombre de classes, cadre de fonctionnement. . . ), ainsi que des thèmes disciplinaires concernés et des objectifs.

La partie 2 permet d'obtenir des informations sur les raisons du choix de l'utilisation des TICE pour le mémoire professionnel. Il s'agit de repérer l'existence d'une prise de conscience des apports des technologies, qui nous semble cruciale pour des usages réussis.

La partie 3 a pour objet de rechercher, dans les mémoires, les traces de pratiques dans le deuxième cadre d'usage des TICE (préparation de la classe) et le troisième (la classe). Certaines questions ont plus particulièrement trait au retour du stagiaire sur sa pratique, et en particulier sur les éléments qu'il a jugés important de relater. Nous espérions également repérer une évolution éventuelle entre le début et la fin des séquences, susceptible de nous fournir des informations sur la genèse des usages des TICE en classe.

La partie 4 s'intéresse à la conclusion du mémoire, qui fait usuellement le point sur les éléments que le stagiaire a considérés comme les plus remarquables au cours de son travail en classe, pour nous permettre de les identifier.

\section{Analyses et résultats}

Les analyses effectuées à l'aide de la grille ont fourni peu de résultats significatifs concernant les mémoires des $\mathrm{PE}$ qui sont par ailleurs très minoritaires 
"abboud" — 2008/9/1 — 18:51 — page 196 — \#10

dans notre échantillon. De ce fait, les résultats que nous présentons dans la suite concerneront uniquement les mémoires des PLC. Cependant, nous soulignons ici un résultat basé sur la comparaison des mémoires PE et PLC : le type d'utilisation de l'ordinateur en classe varie selon le niveau scolaire concerné. En effet, l'utilisation d'un seul poste est limitée, pour notre corpus, à l'école primaire, ce qui est sans doute lié à l'équipement informatique des établissements. A contrario, l'enseignement secondaire ne semble considérer que la seule utilisation de l'ordinateur en salle informatique ou en salle de travaux pratiques ${ }^{8}$ : aucun mémoire ne fait, par exemple, référence à l'utilisation par l'enseignant de l'ordinateur comme tableau interactif.

D'autre part, nous présenterons ici nos résultats en nous attachant à mettre principalement en évidence les régularités qui, comme nous l'espérions, sont apparues à la suite de l'analyse des mémoires et nous semblent donc constituer des traces fiables des usages dans le cadre de la préparation de la classe et dans celui de la classe. Nous avons organisé cette présentation autour de trois axes :

- le premier est lié aux conceptions relatives à l'usage des TICE construites par les stagiaires au cours de leur année de stage;

- le deuxième et le troisième correspondent respectivement aux usages effectifs et aux retours réflexifs sur ces usages qui sont susceptibles de faire naître des questionnements. Certains d'entre eux pourront constituer un levier pour le développement des pratiques, tandis que d'autres se traduiront en termes de résistances à des usages cohérents (Lenfant, 2002).

Les priorités mises en avant par les stagiaires pour intégrer les TICE à leurs pratiques

L'analyse des réponses à la partie 2 de la grille nous renseigne d'une part sur les raisons mises en avant par les stagiaires pour l'intégration des TICE dans leur enseignement, et d'autre part sur les questions qu'il leur semble important de traiter dans le cadre d'une interrogation professionnelle sur cette intégration. Les raisons qui poussent les professeurs stagiaires à utiliser les TICE en classe apparaissent très liées aux apports relatifs à la discipline et relativement peu à l'initiation informatique. Ce résultat est contraire aux représentations obtenues à travers l'étude des questionnaires. Nous trouvons ces apports dans des situations où les technologies font partie intégrante du thème disciplinaire, en référence aux

8 En SVT (Sciences de la Vie et de la Terre), une salle de travaux pratiques standard est constituée de 8 postes de travail élèves et d'un poste professeur. 
programmes en vigueur. C'est bien entendu le cas lorsque l'outil informatique fait l'objet de l'apprentissage, comme le travail sur les tableurs en section STT (Sciences et Technologies Tertiaires ${ }^{9}$ ) et en technologie, ou l'initiation à l'informatique dans le cadre du B2i. C'est également le cas lorsque cet outil est rendu indispensable pour l'approche d'un concept disciplinaire en raison de diverses contraintes pratiques et/ou temporelles, comme pour l'ExAO (Expérimentation Assistée par Ordinateur) en SVT ou l'apprentissage de certains concepts statistiques en mathématiques. Il existe également des situations pour lesquelles l'usage des TICE améliore et favorise le travail dans la discipline; en mathématiques, ceci concerne en particulier les logiciels de géométrie dynamique, qui introduisent une dimension expérimentale et permettent une meilleure visualisation des situations géométriques. Enfin, une dernière raison qui conduit les professeurs stagiaires à utiliser des TICE est que ces technologies leur apparaissent comme un outil attrayant pour les élèves et "confortable" pour l'enseignant (rapidité de traitement, meilleure illustration...). Dans ce cas, l'intervention des TICE n'est pas perçue comme apportant des modifications fondamentales par rapport à l'environnement papier/crayon. Ces résultats confirment ceux de Caliskan et Erdogan qui, dans une étude quantitative portant une sélection de 582 mémoires de PLC2 en mathématiques [10], montrent que les types de TICE privilégiés par les stagiaires correspondent aux usages les plus écologiquement viables [11], c'est-à-dire ceux qui, en mathématiques, peuvent le plus facilement s'intégrer dans une pratique d'enseignement peu modifiée.

L'étude des problématiques déclarées dans les mémoires fait apparaître trois orientations principales quant aux hypothèses/interrogations des stagiaires liées à l'intégration des TICE.

La première, de loin la plus fréquente, est centrée sur leurs apports pour les apprentissages des élèves (par exemple : acquisition de compétences dans l'utilisation du tableur ou meilleure compréhension de la notion de fonction).

La deuxième orientation concerne des interrogations sur l'intégration des TICE, en particulier sur les types de situations à construire ou sur les potentialités et les limites par rapport aux concepts étudiés. On trouve par exemple les questions suivantes posées dans un mémoire du thème D (algèbre en liaison avec la géométrie) : "En quoi l'informatique permet-elle une approche pertinente des objets mathématiques étudiés? Comment construire des séances guidées pour faire face à l'inégalité des savoir-faire et à la difficulté d'utilisation du logiciel?". Cette

\footnotetext{
${ }^{9}$ Tertiaire : bureautique et secrétariat.
} 
orientation se trouve essentiellement dans les mémoires des thèmes B (géométrie) et $\mathrm{C}$ (statistiques), mais elle est également présente dans certains mémoires relatifs au thème $\mathrm{F}$ (initiation informatique).

La troisième orientation, plus rare et généralement couplée avec la précédente, est relative à des interrogations sur les pratiques de l'enseignant pendant les séances intégrant les TICE. En résumé, les problématiques déclarées sont donc largement centrées sur les élèves et leurs apprentissages, et on trouve relativement peu d'interrogations sur les particularités liées à l'intégration des TICE, que ce soit dans l'approche disciplinaire ou dans la pratique de l'enseignant. Cette caractéristique avait également été mise en évidence dans l'étude de Caliskan et Erdogan (op. cit.) qui a distingué deux grandes classes de problématiques : la première, numériquement la plus importante, porte sur des motivations générales à l'usage des TICE (apports et mise en œuvre, possibilités et limites, influence sur la motivation des élèves); tandis que la seconde se centre sur des objectifs d'apprentissage méthodologiques ou notionnels.

Les problématiques apparaissent donc comme principalement centrées sur les apprentissages et plus généralement sur les élèves; on n'y trouve que rarement une réflexion a priori sur les changements qu'induit l'intégration des technologies dans l'apprentissage et l'enseignement.

Les usages réels dans la préparation et la mise en œuvre des séances TICE

L'analyse des réponses à la partie 3 de la grille nous renseigne, d'une part sur la préparation des séances TICE analysées dans les mémoires, d'autre part sur l'activité en classe.

En ce qui concerne la préparation des séances, nous avons observé que la tâche des élèves est très majoritairement préparée sous la forme d'un document écrit distribué en début de séance. Ce document-élève contient souvent, en plus des questions liées aux concepts disciplinaires abordés (du type rencontré en environnement papier-crayon), des indications pour la manipulation du logiciel. L'explication de cette particularité est sans doute à rechercher du côté du thème de la séquence qui, s'il ne consiste pas en la découverte d'un nouveau logiciel, inclut souvent, dans le cadre d'un apprentissage disciplinaire, une utilisation d'un logiciel peu ou pas connu des élèves (ce qui est souvent le cas en SVT).

Quant au degré d'autonomie de l'élève et son évolution, on constate que le document est très souvent directif au départ et que, si l'élève est parfois guidé de bout en bout, il peut aussi arriver que le professeur lui concède davantage d'autonomie s'il le juge profitable. Inversement, lorsque le document laisse l'élève 
"abboud" — 2008/9/1 — 18:51 — page 199 — \#13

autonome au départ, on remarque que, dans la moitié des cas, la gestion de la classe évolue ensuite vers plus de guidage. Si l'on croise maintenant cette dernière constatation avec les résultats concernant l'analyse a posteriori faite par le stagiaire, on se rend compte que ce dernier s'estime contraint de renforcer le guidage par le comportement des élèves, qui perdent de vue l'objectif disciplinaire ou rencontrent des difficultés à accomplir une tâche trop "ouverte".

Enfin, d'une manière générale, mis à part les instructions relatives à la manipulation du logiciel, le document-élève, lorsqu'il existe, ne se distingue pas de ce qu'on peut trouver dans le cas d'un environnement papier/crayon.

En ce qui concerne l'activité en classe, nous avons noté précédemment que la réflexion du stagiaire sur sa propre activité n'apparaît que relativement peu dans la problématique déclarée. Les comptes rendus de séquences font, quant à eux, apparaître l'activité de l'enseignant comme marginale : distribution du document-élève, aide à la prise en main du logiciel, explicitation de la consigne... $\mathrm{Au}$ cours de la phase de travail personnel des élèves, elle se réduit généralement à une aide individuelle ou à un contrôle de l'avancée du travail. Nous faisons l'hypothèse que cette position en retrait du professeur résulte - au moins partiellement - du caractère guidé des tâches assignées à l'élève. Il est également possible que ces enseignants débutants dévoluent une grande partie de leur rôle à l'ensemble document/ordinateur, considéré comme l'interlocuteur privilégié de l'élève. La phase de bilan collectif n'a été que rarement rapportée ou analysée; certains stagiaires évoquent un bilan ultérieur, mais sans donner plus de précisions. On peut, dans une certaine mesure, rattacher ce fait à des problèmes rencontrés dans la gestion du temps, mais on peut également émettre l'hypothèse d'un manque d'intérêt des stagiaires pour cette phase. Ceci ne nous semble pas spécifique aux environnements TICE, surtout pour des enseignants débutants qui se focalisent sur les phases de lancement et d'accompagnement de l'activité des élèves et accordent moins d'importance aux phases de mise en commun, l'essentiel étant pour eux que les élèves accomplissent les tâches prévues, considérées comme auto-suffisantes pour l'apprentissage. Dans certains mémoires est mentionnée une correction collective qui s'est déroulée, soit dans la salle informatique à l'issue du travail sur les ordinateurs, soit ultérieurement en environnement papier/crayon. Ce résultat est, à nouveau, en contradiction avec certains résultats des questionnaires relatifs aux apports "culturels" des technologies.

D'une manière générale, peu d'évolutions ont été notées dans les séquences analysées par les professeurs stagiaires : les seules informations que nous avons pu recueillir concernent en priorité des modifications à apporter aux supports, 
"abboud" — 2008/9/1 — 18:51 — page 200 — \#14

comme "diminuer la quantité d'informations fournie aux élèves" ou l'utilisation d'un "diaporama expliquant certaines manières de faire pour étirer une formule". Ce fait peut être lié aux contraintes de la réalisation du mémoire et, en particulier, au fait qu'il correspond à un temps d'enseignement relativement court (pour notre corpus, moins de 3 heures en moyenne pour une séquence). Dans ces conditions, il est évidemment difficile de repérer une éventuelle évolution.

\section{Les retours des stagiaires sur leur pratique}

Nous avons déjà noté que les stagiaires ne s'interrogent guère sur les particularités de l'intégration des TICE avant la mise en œuvre de leur séquence. En revanche, les analyses a posteriori présentes dans les mémoires font presque toujours apparaître, suite à des difficultés rencontrées dans le déroulement de la séquence, une certaine prise de conscience des spécificités liées à l'utilisation des technologies.

En ce qui concerne la préparation des séquences, des difficultés liées à la longueur du temps de préparation sont notées par plusieurs stagiaires (notamment dans les mémoires des thèmes $\mathrm{A}$ et B). Certains ont également repéré que cette préparation nécessite une anticipation de l'activité de l'élève ainsi qu'une articulation entre les activités en environnement TICE et les activités hors TICE.

En ce qui concerne le déroulement des séquences, l'analyse que font les professeurs stagiaires fait apparaître quelques retours positifs, peu nombreux, qui reviennent sur les dimensions générales des problématiques : la motivation et l'intérêt des élèves pour ce type de travail, une plus grande autonomie des élèves favorisant l'aide individualisée. En revanche, des stagiaires pointent que de nombreux élèves perdent le sens de la tâche qui leur est proposée, du fait que leur attention est surtout accaparée par la manipulation du logiciel et l'affichage à l'écran. Certains signalent la difficulté, pour l'enseignant, à trouver un équilibre entre des activités guidées, où tout se passe bien mais au cours desquelles l'apprentissage des élèves est réduit, et des activités plus ouvertes qui risquent de les décourager. Ceci rejoint les analyses du document-élève que nous avons présentées précédemment.

Par ailleurs, il ressort de manière générale que les stagiaires ont été confrontés à des problèmes liés à la gestion du temps (ceci a été noté dans pratiquement tous les mémoires, à l'exception de ceux relatifs à l'initiation informatique).

D'autres difficultés sont signalées, en particulier du côté des élèves, l'existence de problèmes techniques liés à la manipulation du logiciel et l'observation de "détournements", non prévus par les stagiaires, qui s'opèrent sur les objectifs 
d'apprentissage. En effet, par rapport à un environnement papier/crayon, l'introduction d'un outil TICE implique un nouveau rapport aux objets d'apprentissage. Or, l'ensemble des mémoires analysés témoigne d'une non prise en compte a priori de ce changement; cette prise de conscience, qui n'intervient qu'en cours de séquence, est jugée préoccupante par le stagiaire, ce qui explique peut-être sa présence massive dans les analyses a posteriori. Ainsi, plusieurs mémoires du thème $\mathrm{B}$ (géométrie) mentionnent que l'utilisation d'un logiciel de géométrie dynamique "incite les élèves à se contenter de preuves expérimentales, [et qu'] il est difficile de les ramener à une rigueur théorique". De même, certains mémoires du thème $\mathrm{C}$ (statistique) signalent "la perte de vue du sens des opérations effectuées" lors de l'utilisation du tableur.

On voit aussi apparaître, dans les conclusions des mémoires, une prise de conscience a posteriori du rôle particulier de l'enseignant dans ce genre de séances : il n'est plus le seul détenteur du savoir à enseigner, il se trouve mieux à même de repérer les difficultés des élèves et d'y remédier (éventuellement en apportant une aide individualisée) ; mais il lui est plus difficile de gérer la séance, de contrôler le travail de l'ensemble des élèves et de l'évaluer. Dans les mémoires relevant $\mathrm{du}$ thème $\mathrm{F}$, on trouve une réflexion plus approfondie sur les formes de travail associées aux TICE (travail autonome, par binômes, en groupe). Il y est noté qu'une certaine lassitude des élèves apparaît à la longue, lorsque l'apprentissage "technique" est déconnecté de tâches disciplinaires.

Par ailleurs, les retours réflexifs des stagiaires sur les séances TICE menées peuvent témoigner au moins partiellement des représentations des tuteurs de mémoires relatives à l'intégration des TICE dans l'enseignement de la discipline. Bailleul et Bodergat [6] ont déterminé que, pour être à même de suivre un mémoire professionnel, ces tuteurs doivent avoir suffisamment d'expérience professionnelle et d'informations sur les pratiques habituelles ou innovantes afin de les mettre à la disposition des professeurs stagiaires et doivent savoir les questionner pour les amener à prendre du recul. La rareté des traces d'une réflexion de fond sur les spécificités induites par l'intégration de l'ordinateur dans la classe nous semble ainsi en partie liée à l'état de la réflexion des tuteurs de mémoire sur ce sujet et à l'importance qu'ils lui accordent. Ceci nous renvoie à une question qui reste, à l'heure actuelle, sans réponse pour la recherche, celle de l'influence que peut avoir la genèse des usages des TICE en formation sur la genèse des usages dans l'enseignement ultérieur.
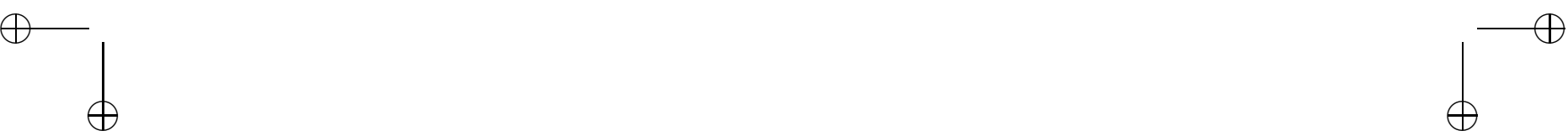
"abboud" — 2008/9/1 — 18:51 — page 202 — \#16

\section{Discussion et perspectives}

Le but de notre recherche était d'approcher les pratiques réelles des enseignants stagiaires dans le domaine de l'intégration des TICE, à travers l'étude de traces de ces pratiques rapportées dans les mémoires professionnels. Nous voulions également tester la validité de certains résultats issus de l'étude par questionnaires, déclarations de stagiaires, en les confrontant à la réalité des pratiques.

Pour commencer, et comme nous l'avons déjà noté, le type d'utilisation des TICE varie selon le niveau scolaire. Cette caractéristique de l'intégration des TICE par les professeurs stagiaires trouve bien sûr son explication dans les contraintes matérielles des établissements scolaires [17], qui limitent de manière évidente les marges de manœuvre des enseignants [29]. Mais on peut également l'expliquer par le fait que les schèmes d'utilisation des artefacts par les enseignants semblent résulter en partie de l'appropriation de schèmes professionnels préexistants [18]. Or, il semble actuellement que peu de professeurs intègrent réellement les outils informatisés dans leurs démarches professionnelles [25]. Il n'est donc pas étonnant que la mise en place de pratiques innovantes soit rare à l'issue de l'année de stage.

Notre recherche tend également à montrer que dans leurs mémoires professionnels les stagiaires ne se centrent pas uniquement sur les apports liés à l'initiation aux technologies. En effet, il s'avère que les priorités mises en avant par les stagiaires pour utiliser les TICE sont majoritairement centrées sur les apports aux apprentissages disciplinaires des élèves. Ceci peut s'expliquer à la fois par les injonctions des programmes scolaires et par le fait qu'institutionnellement le mémoire professionnel doit porter sur l'enseignement effectif du stagiaire et se trouve donc en étroite relation avec les apprentissages des élèves.

Par ailleurs, il s'avère que, même si les professeurs stagiaires s'interrogent peu a priori sur les spécificités de l'intégration d'outils informatiques lors des séances, les difficultés qu'ils rencontrent au cours de la mise en oeuvre de telles séances les conduisent à pointer et à analyser certains problèmes rencontrés. Cette caractéristique rejoint les résultats de travaux comme ceux de Lenfant (op. cit.), relatifs à la construction de compétences professionnelles, où l'on voit, par exemple, des professeurs stagiaires confrontés à certaines difficultés d'élèves passer, au cours de leur année de stage, de constats et d'explications répandues, mais sommaires, à la construction de systèmes explicatifs plus élaborés. Cette caractéristique apparaît donc comme un point crucial dans la genèse des schèmes d'utilisation associés aux technologies dans l'activité des enseignants débutants. 


$$
\text { "abboud" — 2008/9/1 — 18:51 — page } 203 \text { — \#17 }
$$

En revanche, la prise de conscience a posteriori portant sur le rôle particulier de l'enseignant et sur les spécificités de l'intégration des TICE ne conduit pas majoritairement les stagiaires à des propositions de transformation de leur séquence ; les difficultés repérées sont vues comme liées à une innovation inhérente à l'outil, sans qu'une évolution vers un usage plus satisfaisant soit envisagée.

Enfin, l'absence de phases collectives et notamment de bilan, rejoint des observations souvent faites en formation lors de visites de stagiaires ou dans les mémoires professionnels, quel que soit l'environnement (TICE ou non). Ceci est révélateur d'un des points de résistance dans la construction des pratiques enseignantes, fondé sur l'idée que l'activité de l'élève suffit à assurer l'apprentissage.

Aujourd'hui nous sommes dans une période où les stagiaires intégrant l'IUFM sont très majoritairement équipés en matériel informatique personnel, et possèdent aussi, en principe, le C2i niveau 1 et donc les compétences relatives à une utilisation personnelle des nouvelles technologies (cadres d'usages 1 et 2). La période actuelle est également celle de la généralisation du C2i niveau 2 enseignant dans laquelle l'IUFM est amené à jouer un rôle plus conséquent dans la formation initiale des stagiaires vis-à-vis des utilisations effectives des TICE dans les classes. Pourtant, à travers notre étude des mémoires professionnels, nous avons souligné plusieurs aspects des usages des TICE dans les classes qui semblent se conformer aux normes existantes dans l'enseignement disciplinaire "hors TICE" et qui semblent se cantonner au stade de "l'innovation naïve", sans se situer dans une perspective d'usages intégrés à la pratique [3].

La suite de notre travail s'oriente actuellement vers une étude plus approfondie du rôle des dispositifs de formation dans les IUFM dans l'évolution de cette première intégration "naïve" des technologies vers une intégration plus réfléchie prenant en compte leurs spécificités. Une question reste cependant ouverte : où et comment ces enseignants débutants vont-ils rencontrer des modèles de pratiques TICE qu'ils pourraient considérer comme faisant partie des normes de leur métier?

\section{Annexe : GRILLE D'ÉTUDE DES MÉMOIRES}

Remarques préliminaires :

Les rubriques I et III peuvent être, pour un même mémoire, dupliquées si besoin (plusieurs niveaux de classes, outils différents, thèmes différents... ). 
Lorsque les questions portent sur des contenus disciplinaires, il s'agit d'y répondre de façon précise, même si l'on aborde des notions spécifiques à la discipline et à sa didactique (une synthèse en sera faite ultérieurement).

\section{INFORMATIONS GÉNÉRALES}

1.1. Nom de(s) l'outil(s) informatique(s)

1.2. Niveau(x) concerné(s)

1.3. Nombre de classes

1.4. Séquence étudiée (nombre de séances, durée, insertion dans la progression...)

1.5. Durée d'utilisation de l'outil informatique dans la séquence et/ou la séance

1.6. Thème(s) disciplinaire(s) (s'il y a lieu) et objectif(s) relatif(s) au(x) thème $(\mathrm{s})$

1.7. Type(s) de fonctionnement effectif(s) pour la séquence et/ou la séance

1.7.a. poste unique : professeur et/ou élève(s)

1.7.b. plusieurs postes :

- en interne : sans réseau / avec réseau

- liaison Internet

2. SPÉCIFICITÉ DES TICE DANS LA PROBLÉMATIQUE DU MÉMOIRE (déclaration du stagiaire)

2.1. Quelle est la problématique déclarée?

2.2. Quelles sont les raisons générales invoquées par le stagiaire pour justifier l'utilisation des TICE dans la séquence/séance?

2.2.a. Pas de raisons déclarées car les outils TICE sont culturellement utilisés dans la discipline

2.2.b. Institutionnelles

2.2.c. Autres (à préciser)

2.3. Y a-t-il des apports spécifiques, relatifs aux contenus disciplinaires, de l'usage des TICE dans les situations d'apprentissage étudiées? Si oui, lesquels?

2.4. Y a-t-il des apports spécifiques, relatifs à l'initiation informatique des élèves à l'usage des TICE dans les situations d'apprentissage étudiées? Si oui, lesquels? (Préciser notamment si l'objectif de certification (B2i) est explicite ou non.) 
"abboud" — 2008/9/1 — 18:51 — page 205 — \#19

Comment les enseignants en formation initiale utilisent l'informatique en classe

205

2.5. Y a-t-il réflexion a priori sur la spécificité du rôle de l'enseignant en environnement TICE? Si oui laquelle?

\section{LA (LES) SÉANCE(S) EFFECTIVE(S)}

3.1. La préparation des tâches des élèves

3.1.a. Sources de l'activité (manuel, document pédagogique, liste de diffusion, création/adaptation personnelle...)

3.1.b. - Nature du document élève (s'il existe) :

- contient-il des aides à la "manipulation" de l'ordinateur?

- contient-il des questions relatives $\mathrm{au}(\mathrm{x})$ thème(s) disciplinaire(s)?

- Quel est le degré d'autonomie de l'élève par rapport à la (aux) consigne(s) donnée(s) dans le document? Y constate-t-on une évolution dans la forme du travail demandé à l'élève?

3.2. Les prévisions de gestion de la classe

3.2.a. Quelles sont les contraintes (si elles existent) que le stagiaire a identifiées?

3.2.b. Quels sont les choix effectués par le stagiaire?

3.3. L'activité du stagiaire pendant les séances.

A-t-il rencontré des difficultés liées au TICE? Si oui :

- à quoi étaient-elles dues?

- comment les a-t-il gérées?

3.4. Le moment du bilan collectif (s'il a eu lieu, même s'il est effectué en dehors des séances TICE).

Prend-il en compte des éléments spécifiques aux TICE? Si oui, précisez.

3.5. L'analyse a posteriori de la séance.

Quels retours critiques le stagiaire fait-il sur la séance?

- retours positifs

- retours négatifs

\section{CONCLUSION DU MÉMOIRE}

Précisez les éléments (voir rubrique 2) sur lesquels le stagiaire fait un retour critique dans sa conclusion. 


\section{Références}

[1] M. Abboud-Blanchard, Réflexions sur la formation des enseignants à l'utilisation de logiciels dans leur enseignement, in: Faire des mathématiques avec un système de calcul formel, CNDP Paris, 1998, 137-154.

[2] M. Abboud-Blanchard, How mathematics teachers handle lessons in technology environments, Proceedings of the $5^{\text {th }}$ Nordic Conference on Research in Mathematics Education, Copenhagen, Denmark (2008).

[3] M. Abboud-Blanchard and JB. Lagrange, Uses of ICT by pre-service teachers : towards a professional instrumentation?, International Journal for Technology in Mathematics Education 13, no. 4 (2006), 183-190.

[4] M. Artigue, Learning mathematics in a CAS environment : the genesis of a reflection obout instrumentation and the dialectics between technical and conceptual work, International Journal of Computers for Mathematical learning 7 (2002), 245-274.

[5] G. Baillat et J. Vincent, Les TICE et les jeunes enseignants : les représentations en formation initiale, in: Actes du colloque européen ITEM 2003, Reims, 2003.

[6] M. Bailleul et J. Y. Bodergat, Diriger un mémoire professionnel. Entre dispositif institutionnel et problématique relationnelle. Le cas de la formation des enseignants en France, European Journal of Teachers Education 24, no. 3 (2001), 263-289.

[7] G.-L. Baron, Les TICE, de l'innovation à la scolarisation : problèmes et perspectives. Transcription de l'intervention effectuée le 15/01/2005 dans le colloque AFT "Accompagner les TICE à l'école", 2005,

http://aft-rn.net/actes_colloque05/conferences/conference_GL_Baron.pdf.

[8] G.-L. Baron et E. Bruillard, Technologies de l'information et de la communication : quelles compétences pour les enseignants?, Education et formations 56 (2000), $153-159$.

[9] D. Butlen, A. Dubut, P. Masselot, B. Ngono, ML. Peltier and M. Pezard, Pratiques de professeurs d'école enseignant les mathématiques en réseaux d'éducation prioritaire : cohérence et contradictions, Rapport de recherche, IREM de l'université Paris 7 (2003).

[10] N. Caliskan and E. Erdogan, La place des TICE dans les mémoires professionnels d'IUFM, in: Actes du colloque européen ITEM 2003, Reims, 2003.

[11] Y. Chevallard, Intégration et viabilité des objets informatiques dans l'enseignement des mathématiques, in: L'ordinateur pour enseigner les Mathématiques, Nouvelle Encyclopédie Diderot, PUF, Paris, 1992, 183-203.

[12] C. Comiti, S. Nadot et E. Saltiel, Le mémoire professionnel : enquête sur un outil de formation des enseignants, IUFM de Grenoble, 1999.

[13] J. P. Da Ponte, H. Oliveira et J. M. Varandas, Development of pre-service mathematics teachers' professional knowledge and identity in working with information and communication technology, Journal of Mathematics Teacher Education 5 (2002), 93-115. 
"abboud" — 2008/9/1 — 18:51 — page 207 — \#21

[14] DGES, Direction Générale de l'Enseignement Scolaire, Les programmes scolaires (de l'école primaire, du collège et des lycées), 2005, http://eduscol.education.fr/D0048/LLPPRC01.htm.

[15] EducnetB2I, B2I, Brevet Informatique et Internet, 2007, http://www2. educnet. education.fr/sections/formation/certification/b2i/.

[16] EducnetC2I, C2I, Certificat Informatique et Internet. Niveau 2 "enseignant", 2007, http://www2.educnet. education.fr/sections/formation/certification/c2i8908/.

[17] ETIC, Enquête sur les Technologies de l'Information et de la Communication, Enquête menée par la DT-SDTICE (Direction de la Technologie) et la DEP (Direction de l'Evaluation et la Prospective) : équipements et usages des TIC dans les écoles, collèges et lycées, 2005, http://www2.educnet.education.fr/sections/plan/etic/.

[18] R. Goigoux, Contribution de la psychologie ergonomique au développement de la didactique du français, in: Balises en didactique des mathématiques, Grenoble, La pensée sauvage, 2005, 17-39.

[19] T. Karsenti, Impact des TIC sur l'attitude, la motivation et le changement dans les pratiques pédagogiques des futurs enseignants, in: La profession d'enseignant aujourd'hui, (M. Tardif, C. Lessard, eds.), Ed. De Boeck, 2004, 187-205.

[20] J. B. Lagrange, M. Artigue, C. Laborde and L. Trouche, Technology and mathematics education : a multidimensional study of the evolution of research and innovation, in: the Second International Handbook of Mathematics Education, (A. J. Bishop, M. A. Clements, eds.), 2003, 239-271.

[21] J. B. Lagrange, J. F. Lecat et B. Parzysz, Les professeurs stagiaires d'IUFM et les technologies, quelle instrumentation ?, Recherche et Formation 52 (2006), 131-146.

[22] P. Le Borgne, J.-P. Fallot, J.-F. Lecas et A. Lenfant, Usages des technologies par les élèves professeurs : analyse à partir de questionnaires, Revue internationale des technologies en pédagogie universitaire 2, no. 3 (2005), 7-14.

[23] A. Lenfant, De la position d'étudiant à la position d'enseignant : l'évolution du rapport à l'algèbre de professeurs stagiaires, Université Paris 7, 2002.

[24] J. Monaghan, Teachers' activities in technology-based mathematics lessons, The International Journal of computers for mathematical learning 9, 2004, 327-357.

[25] OCDE, Organisation de Coopération et de Développement Economiques, Completing the Foundation for Lifelong Learning : An OECD Survey of Upper Secondary Schools, 2004,

http://www.oecd.org/document/18/0, 2340,fr_2649_201185_26304850_1_1_1_1,00.html.

[26] L. Paquay, M. Altet, E. Charlier et P. Perrenoud, Former des enseignants professionnels. Quelles stratégies? Quelles compétences?, Ed. De Boeck, Paris, Bruxelles, 1996.

[27] P. Perrenoud, La formation des enseignants entre théorie et pratique, Ed. L'Harmattan, Paris, 1995.

[28] R. Rabardel, Les hommes et les technologies. Approche cognitive des instruments contemporains, Ed. Armand Colin, 1995. 
[29] A. Robert and J. Rogalski, Le système complexe et cohérent des pratiques des enseignants de mathématiques : une double approche, La revue canadienne des sciences, des mathématiques et des technologies 2, no. 4 (2002), 505-528.

[30] JM. Van Der Maren, En quête d'une recherche pratique, Sciences Humaines 142 (2003), 42-44.

MAHA ABBOUD-BLANCHARD

ÉQUIPE DIDIREM UNIVERSITÉ PARIS 7, CASE COURRIER 7018

2, PLACE JUSSIEU, 75251 PARIS CEDEX 05

FRANCE

E-mail: maha.blanchard@math.jussieu.fr

JEAN-PAUL FALLOT

IUFM DE FRANCHE-COMTÉ

FRANCE

E-mail: jean-paul.fallot@fcomte.iufm.fr

AGNÈS LENFANT

ÉQUIPE DIDIREM

FRANCE

E-mail: agnes.lenfant@univ-reims.fr

BERNARD PARZYSZ

ÉQUIPE DIDIREM

FRANCE

E-mail: parzysz.bernard@wanadoo.fr

(Received February, 2008) 